3) The application of the fibrolytic enzyme in the initial stage of the illness is one of the effective treatments.

\title{
105. Intermittent Vertebral Artery Compression
}

-A contribution to the study of the thoracic outlet syndrome-

\author{
Chikao Nagashima, Tsuneo OHono and Norimoto AraI \\ Dept. of Neurosurgery, Kanto Rosai Hospital
}

10 patients of intermittent vertebral artery compression first described by Powers 1961 are discussed with particular reference to diagnosis, correlation with trauma and surgical treatment. Retrograde vertebral angiography confirms the diagnosis by demonstraiting intermittent occlusion upon forced lateral rotation or hyperextension of the head.

Particular stress is placed, first, on oto-neurological examination for diagnosis of brain stem dysfunction due to arterial insufficiency of vertebro-basilar system with electronystagmographic tracing upon rotating or hyperextending the head or upon changing posture. Second, on the supra-clavicular murmur with marked increase of intensity especially upon rotating head with holding deep breath. Third, on X-ray evidence of the cervical rib, elongated transverse process of $\mathrm{C} 7$, and higher arch and sharper curve of subclavian artery in pre-operative angiography. These findings are noted in 5 out of 6 patients with post-traumatic onset. One of authors (C. N.) has a speculation as follows. With the subclavian artery well up into the soft part of the neck and above the clavicle, it runs greater risk of injury from trauma and this anatomical variation might play an important predisposition for development of his sort of syndrome following injury. Surgical treatment carried out in 9 cases gave immediate and lasting relief of symptoms.

\section{Observation of Cerebral Blood Flow in Intracranial Arteriovenous Malformation}

\author{
Shin Hoshikawa, Hajime NagaI, Muneyoshi Kayo, Masahiro Furuse \\ Kazuhiko OKamura, Inazo Toda, Yoshihiko OKa, Ryohei Hayakawa \\ Tatsuya Kobayashi, Atsushi IKeyama, Shigeru MaEda \\ and Michiaki Hasuo \\ 2nd Department of Surgery, Nagoya University School of Medicine
}

By means of two techniques, $\mathrm{Kr} 85$ clearance technique by Lassen et al and 
nondiffusible RI injection technique by Olendorf et al, cerebral circulation was studied in 4 patients with intracranial arteriovenous malformation.

Since the clearance rate of $\mathrm{Kr} 85$ from the brain depends upon the blood flow washing out the radioisotope from the tissue, the value obtained with this technique could represent the 'effective blood flow'; it referes to the blood flow which traverses capillaries and is engaged in the exchange of nutrients and metabolites.

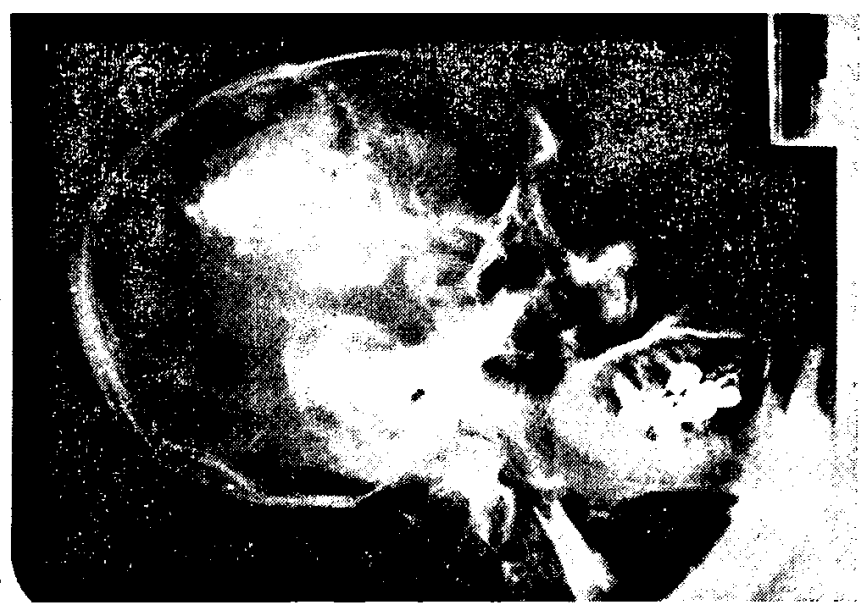

Fig. 1. Angiography demonstrate a large arteriovenous malformation in right parietal area.

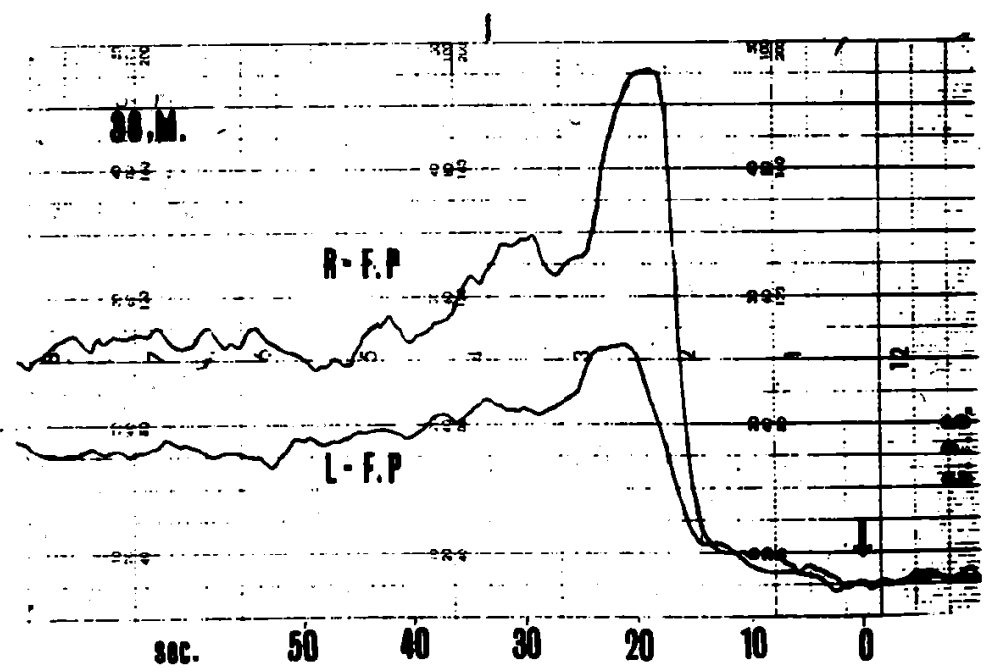

Fig. 2. RI cerebrogram obtained from the lesion shows a high peak and continuing high level compared to that from normal hemisphere, showing the increased blood flow which pass through the creebral vascular bed. 
On the other hand, the dilution curve obtained by external tracing of the radiaactive bolus traversing the brain, reflects the blood flow which passes through the intracranial vascular bed, since nondiflusible indicator (RISA was used in most cases) which does not move across the blood-brain barrier, remains within vessels.

In all of 4 patients, the dilution curve of radioactive bolus obtained from the lesion showed a marked rise in count rate compared with that from the contralateral hemisphere, indicating the increased blood flow through the vascular bed; that could be due to the blood flow which traverses the arteriovenous shunt. (Fig. 2)

On the other hand, a characteristic record was obtained from the lesion by Kr 85 clearance technique; it consisted of a characteristic high peak followed by low clearance curve. (Fig. 3)
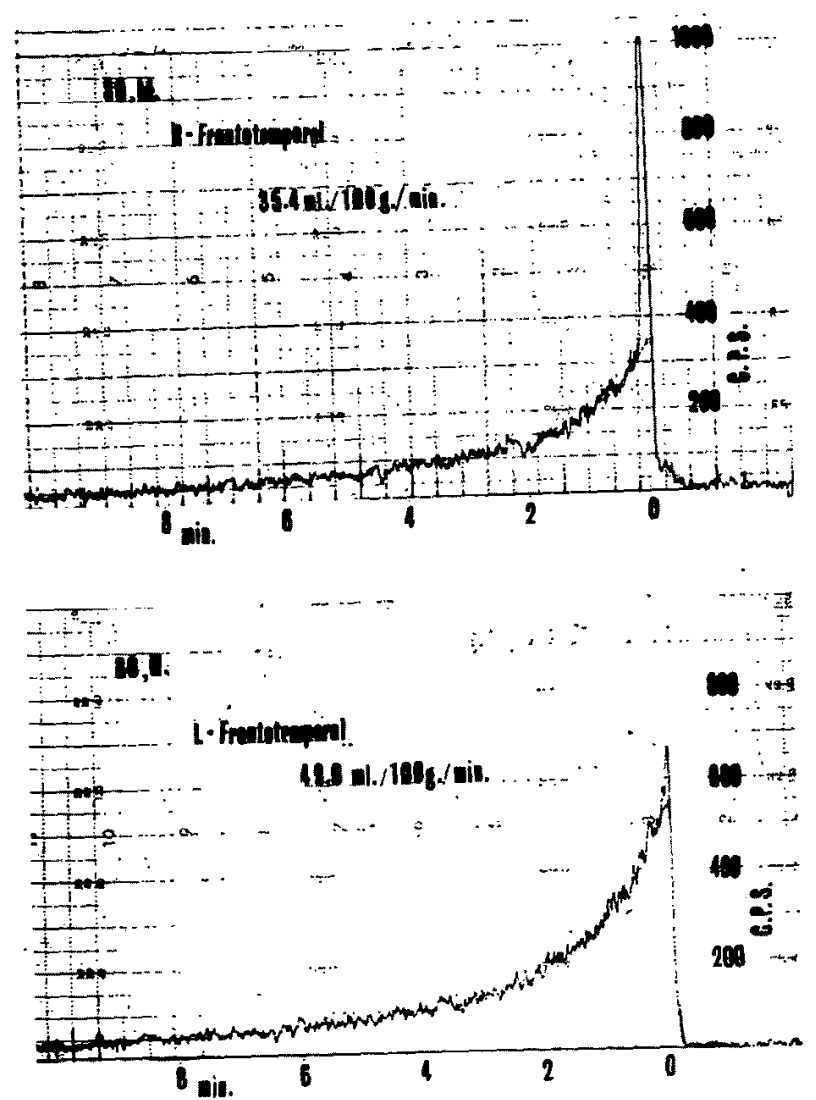

Fig. 3. The clearance curve obtained from the lesion shows a high peak followed by low clearance curve.

This initial peak is considered to reflect the rapid passage of $\mathrm{Kr} 85$ through arteriovenous shunts. 
The values calculated from the clearance curves were as follows: 35.4 $\mathrm{ml} / 100 \mathrm{~g} / \mathrm{min}$. from the lesion and 49.0 from the contralateral hemishere in $1 \mathrm{st}$ case, 33.2 and 45.0 in $2 \mathrm{nd}, 43.5$ and 57.0 in $3 \mathrm{rd}, 52.0$ and 50.7 in 4 th case, respectively.

In 3 patients in whom the draining vessels were demonstrated angiographically, the effective blood flow was decreased in the lesion, while the radioisotope dilution curve showed the markedly increased blood flow passing through the vascular bed.

In the remaining one who was not detected clear draining vessel angiographically, the decrease of effective cerebral blood flow was not observed.

In arteriovenous malformation, the 'effective blood flow' which is involved in the exchange of metabolites, could be decreased due to the blood deprivation by arteriovenous shunt, although the blood flow which pass through the vascular bed is increased due to that through the arteriovenous shunt, while in those with small arteriovenous shunt, the blood deprivation could be small and so the decreased effective blood flow could not be observed.

\title{
107. Diagnostic Application of Dye-Dilution Technics in Cerebral Arteriovenous Aneurysm
}

\author{
Takashi Nakamura, Takashi Kutsuzawa, Satoru Takahashi, \\ Chikahiro SAITo and Sohsuke TAKaHASHI \\ 1st Department of Internal Medicine, Tohoku University School of Medicine
}

A method for measuring a rate of cerebral shunted blood flow in cerebral arteriovenous ancurysm by the dye-dilution method has been evaluated.

Indocyanine green was injected into the right and left common carotid arteries; dye concentration curves of the blood from both junglar bulbs were recorded simultaneously while blood was withdrawn at a constant rate through photoconductive-cell densitometers. The curves were plotted on semilogarithmic paper and the downslope extrapolated by the method of Hamilton.

Dye-curves of cerebral arteriovenous aneurysm were characterized by two peaks and the reduction of the dye-appearance time.

Rate of shunted blood flow was calculated by the comparison between both areas of the dye concentration in the primary and second wave.

In six cases of cerebral arteriovenous aneurysm, interrelationship of the rate of shunted blood flow measured by the present method, cerebral blood flow determined by the nitrous oxide method and the size of tumor visualized on cerebral angiogram was investigated. 